\title{
Anterior interosseous nerve syndrome
}

\author{
Fascicular motor lesions of median nerve trunk
}

Mirko Pham, MD

Philipp Bäumer, MD

Hans-Michael Meinck, MD

Johannes Schiefer, MD

Markus Weiler, MD

Martin Bendszus, MD

Henrich Kele, MD

Correspondence to

Dr. Pham:

mirko.pham@med.uni-

heidelberg.de

Editorial, page 554

Supplemental data at www.neurology.org

\section{ABSTRACT}

Objective: We sought to determine lesion sites and spatial lesion patterns in spontaneous anterior interosseous nerve syndrome (AINS) with high-resolution magnetic resonance neurography (MRN).

Methods: In 20 patients with AINS and 20 age- and sex-matched controls, MRN of median nerve fascicles was performed at $3 T$ with large longitudinal anatomical coverage (upper arm/elbow/ forearm): 135 contiguous axial slices (T2-weighted: echo time/repetition time 52/7,020 ms, time of acquisition: 15 minutes 48 seconds, in-plane resolution: $0.25 \times 0.25 \mathrm{~mm}$ ). Lesion classification was performed by visual inspection and by quantitative analysis of normalized $\mathrm{T} 2$ signal after segmentation of median nerve voxels.

Results: In all patients and no controls, T2 lesions of individual fascicles were observed within upper arm median nerve trunk and strictly followed a somatotopic/internal topography: affected were those motor fascicles that will form the anterior interosseous nerve further distally while other fascicles were spared. Predominant lesion focus was at a mean distance of $14.6 \pm 5.4$ $\mathrm{cm}$ proximal to the humeroradial joint. Discriminative power of quantitative T2 signal analysis and of qualitative lesion rating was high, with $100 \%$ sensitivity and $100 \%$ specificity $(p<0.0001)$. Fascicular T2 lesion patterns were rated as multifocal $(n=17)$, monofocal $(n=2)$, or indeterminate $(n=1)$ by 2 independent observers with strong agreement (kappa $=0.83$ ).

Conclusion: It has been difficult to prove the existence of fascicular/partial nerve lesions in spontaneous neuropathies using clinical and electrophysiologic findings. With MRN, fascicular lesions with strict somatotopic organization were observed in upper arm median nerve trunks of patients with AINS. Our data strongly support that AINS in the majority of cases is not a surgically treatable entrapment neuropathy but a multifocal mononeuropathy selectively involving, within the main trunk of the median nerve, the motor fascicles that continue distally to form the anterior interosseous nerve. Neurology ${ }^{\circledR}$ 2014;82:598-606

\section{GLOSSARY}

AIN = anterior interosseous nerve; AINS = anterior interosseous nerve syndrome; $\mathbf{A U C}=$ area under the curve; $\mathbf{C l}=$ confidence interval; DTI = diffusion tensor imaging; FDP = flexor digitorum profundus; FDPII = index finger; FDPIII = middle finger; FPL = flexor pollicis longus (thumb); IVIg = IV immunoglobulin; MRC = Medical Research Council; $\mathbf{M R N}=$ magnetic resonance neurography; NCS = nerve conduction studies; $\mathbf{P Q}=$ pronator quadratus (forearm); $\mathbf{R O C}=$ receiver operating characteristic; $\mathbf{R O I}=$ region of interest.

Spontaneous anterior interosseous nerve syndrome (AINS) is an uncommon peripheral neuropathy of unclear etiology. ${ }^{1}$ Except for fine articular branches at the wrist, the anterior interosseous nerve (AIN) is an almost purely motor branch of the median nerve important for thumb and hand function. ${ }^{2}$ It leaves the median nerve trunk at forearm level, immediately distally to the pronatorteres muscle, and innervates the flexor pollicis longus (FPL), pronator quadratus (PQ), and flexor digitorum profundus (FDP) muscle to the index and middle finger. ${ }^{2,3}$ AINS presents with spontaneous acute weakness of distal phalanx flexion of the thumb (FPL) and/or index finger (FDPII), middle finger (FDPIII), and forearm pronation (PQ). The severity and completeness of these motor symptoms vary substantially, as described originally. ${ }^{4}$ Typically, no sensory abnormalities are

From the Departments of Neuroradiology (M.P., P.B., M.B.) and Neurology (H.-M.M., M.W.), Heidelberg University Hospital; Clinical Cooperation Unit Neurooncology (M.W.), German Cancer Research Center (DKFZ), Heidelberg; Department of Neurology (J.S.), RWTH University Hospital Aachen; and Center for Neurology and Clinical Neurophysiology Neuer Wall (H.K.), Hamburg, Germany.

Go to Neurology.org for full disclosures. Funding information and disclosures deemed relevant by the authors, if any, are provided at the end of the article. This is an open access article distributed under the terms of the Creative Commons Attribution-Noncommercial No Derivative 3.0 License, which permits downloading and sharing the work provided it is properly cited. The work cannot be changed in any way or used commercially. 
detected by clinical or electrophysiologic examination. However, pain of different quality, intensity, and location may occur. ${ }^{1,5}$

Usually median nerve conduction studies (NCS) are normal in AINS and thus unhelpful for lesion localization. EMG reveals typical patterns of muscle denervation compatible with a lesion of the AIN itself or, alternatively, of its motor fascicles located further proximally within the median nerve trunk. These fascicles continue distally in an ordered fashion of functional grouping to form the AIN. In fact, a more proximal lesion site has been suggested previously. ${ }^{6,7}$ However, it has been difficult to obtain evidence of a more proximal lesion because NCS/EMG may not differentiate it from a lesion to the AIN itself. This study used high-resolution magnetic resonance neurography (MRN) to determine lesion sites and spatial lesion patterns of AINS and estimated its accuracy in discriminating between AINS and controls.

METHODS Between April 2009 and March 2013, 24 consecutive patients with symptoms of AINS were referred to the Department of Neurology, Heidelberg University Hospital, Germany, or the Center for Neurology and Clinical Neurophysiology Neuer Wall, Hamburg, Germany. Twenty of 24 patients consented to undergo MRN and were scheduled prospectively (figure e-1 on the Neurology ${ }^{\circledR}$ Web site at www.neurology.org). Nonspontaneous AINS following trauma was not included. Twenty age- and sex-matched controls without symptoms or signs of median neuropathy or risk factors for neuropathy such as diabetes, alcoholism, or infectious diseases underwent the same imaging protocol.

Standard protocol approvals, registrations, and patient consents. The study was approved by the institutional ethics committee (S-057/2009). All subjects gave written informed consent to participate.

Clinical and electrophysiologic examination. All patients underwent clinical/electrophysiologic examinations performed by board-certified neurologists with at least 10 years of experience in clinical neurophysiology (H.K. or H.M.M.). Motor strength was recorded for FPL, FDPII, and FDPIII using the Medical Research Council (MRC) rating scale. Complete AINS was defined as weakness (MRC $\leq 4)$ of FPL and FDPII/III. PQ was not evaluated quantitatively. Incomplete AINS was defined as weakness (MRC $\leq 4)$ of FPL or FDPII. In all patients, motor and sensory NCS were performed on ipsilateral median/ulnar nerves and contralateral median nerve with surface stimulation at standard sites including the axilla. ${ }^{8}$ Compound muscle action potentials were recorded following supramaximal stimulation from abductor pollicis brevis and abductor digiti minimi muscle. Sensory nerve action potentials were obtained antidromically over second, third, and fifth digit. Furthermore, median and ulnar nerve F-waves and cortical median somatosensory evoked potentials were measured. Using concentric needle EMG, FPL, PQ, or FDPII was examined for signs of denervation.

MRN. All participants underwent MRN at Heidelberg University Hospital. MRN acquired nerve T2 signal at high spatial resolution and, at the same time, provided large proximal to distal coverage along the upper extremity. Participants were examined prone at 3T magnetic field strength (Magnetom VERIO, Siemens, Erlangen/ Germany) using an 8-channel phased-array extremity coil. Three contiguous slabs were recorded with a $2 \mathrm{D}$ fat-saturated T2-weighted turbo spin echo pulse sequence (echo time/repetition time 52/7,020 ms, time of acquisition 5 minutes 16 seconds, inplane resolution $0.25 \times 0.25 \mathrm{~mm}$, slice thickness $3 \mathrm{~mm}$, interslice gap $0.3 \mathrm{~mm}, 45$ slices). The 3 T MRI combined with the employed extremity coil provided sufficient signal to resolve fascicles within median nerve. At lower field strength, such detail may not be achievable. Slab position was as follows: 1) distally: central forearm to humeroradial joint, 2) centrally: humeroradial joint to central upper arm, 3) proximally: central upper arm to axilla.

Image and statistical data analysis. Images were rated qualitatively with regard to the following items by 2 investigators (M.P., P.B.) blinded to all participant data.

1. Lesion determination: Dichotomous ratings on presence vs absence of lesions as evident by increased T2 signal of median nerve fascicles were obtained independently from both raters.

2. Lesion localization: Anatomical site of predominant lesion focus, i.e., the slice position (with reference to humeroradial joint) with strongest increase in T2 signal of fascicles, was determined by consensus.

3. Fascicular involvement: Dichotomous consensus ratings were obtained on whether increased T2 signal involved the entire nerve cross-section or only a partial area of nerve cross-section (fascicular lesion).

4. Longitudinal lesion pattern: Dichotomous ratings were obtained independently from both raters on multifocality vs monofocality. Multifocality: slices with increased fascicular T2 signal alternated with normal slices. Monofocality: single lesion focus over contiguous slices with normal T2 signal proximal and distal to it.

Further steps of quantitative analysis were undertaken. In controls, a region of interest (ROI) was defined by manual segmentation of the median nerve $\left(\mathrm{ROI}_{\text {median_control }}\right)$. $\mathrm{ROI}_{\text {median_control was }}$ derived in each control at $14.6 \mathrm{~cm}$ (tolerance of $\pm 0.6 \mathrm{~cm}$ ) proximal to the humeroradial joint. This distance corresponded to the mean distance in patients of the predominant lesion focus proximal to the humeroradial joint $(14.6 \pm 5.4 \mathrm{~cm}$, table e-1).

In patients, the median nerve was segmented on the slice harboring the predominant lesion focus. Two ROIs within the segmented median nerve were defined: 1) ROI $\mathrm{I}_{\text {median_lesion }}$ comprised the area within nerve cross-section at the dorsal and radial/lateral aspect consistently showing increased T2 signal. 2) ROI median_no_lesion comprised the remainder of nerve cross-section excluding $\mathrm{ROI}_{\text {median_lesion }}$. For each subject, the mean T2 signal of the medial head of the biceps muscle was determined to calculate normalized median nerve T2 values as follows:

Control subjects:

$$
\mathrm{T} 2_{\text {median_control }}=\mathrm{ROI}_{\text {median_control }} / \mathrm{ROI}_{\text {muscle }}
$$

Patients (lesioned fascicles):

$$
\mathrm{T} 2_{\text {median_no_lesion }}=\mathrm{ROI}_{\text {median_no_lesion }} / \mathrm{ROI}_{\text {muscle }}
$$

Patients (normal-appearing fascicles):

$$
\mathrm{T} 2_{\text {median_lesion }}=\mathrm{ROI}_{\text {median_lesion }} / \mathrm{ROI}_{\text {muscle }}
$$

Discriminative power was then evaluated by calculating sensitivity and specificity for qualitative and quantitative data. To objectify the average lesion focus on cross-section, intersubject image registration was performed with 6 degrees of freedom 
(FMRIB Software Library v 5.0). ${ }^{9}$ Before registration, images were flipped horizontally from right to left in patients with right sided symptoms to obtain comparability with patients affected contralaterally. Then, spatial maps of mean normalized T2 values were rendered for patients. Statistical analyses were calculated with STATA 12 (StataCorp LP, College Station, TX).

RESULTS Clinical findings. Mean age of patients was $46.4 \pm 11.1$ years (15 male/5 female) and $45.3 \pm$ 11.3 in controls (15 male/5 female). Mean duration between symptom onset and clinical/electrophysiologic examinations was 22 days (range 2-94) and 3.4 months (range 12 days to 9 months) between symptom onset and MRN. Detailed findings are given in table e-1. Complete AINS was observed in 15 and incomplete AINS in 5 patients. Sensory testing, sensory and motor NCS including F-waves, evoked potentials, and EMG of biceps/triceps were normal in all patients. EMG of FPL, FDPII, or PQ showed denervation in all patients. Pain before or at symptom onset was reported by 13 of 20 patients and varied with regard to quality, anatomical distribution, and time of onset relative to onset of motor symptoms. In the majority of these patients, pain was experienced as sharp or burning sensation at the medial aspect of elbow or upper arm.

Imaging findings and statistical image analysis. Figure 1 illustrates a complete array of T2 source images showing the predominant lesion focus of each patient.

Lesion determination and localization. T2 lesions of the median nerve were rated as present in all patients and absent in all controls (sensitivity 100\%, specificity $100 \%$, interrater agreement: Cohen kappa $=1$ ). The positions of predominant lesion foci are given in table e-1. Their spatial distribution is illustrated in figure 1. Mean distance of predominant lesion focus was $14.6 \pm 5.4 \mathrm{~cm}$ proximal to the humeroradial joint.

Fascicular involvement. In all patients but none of the controls, median nerve T2 lesions were present and involved only a partial area of the nerve cross-section (Cohen kappa $=1)$. The exact location of T2 signal increase was at the dorsal and radial/lateral aspect of the nerve cross-section. As illustrated on a somatotopic map of median nerve fascicles (figure 2), this lesion area corresponded precisely to the somatotopic/

Figure 1 Fascicular lesions of median nerve at upper arm level in anterior interosseous nerve syndrome

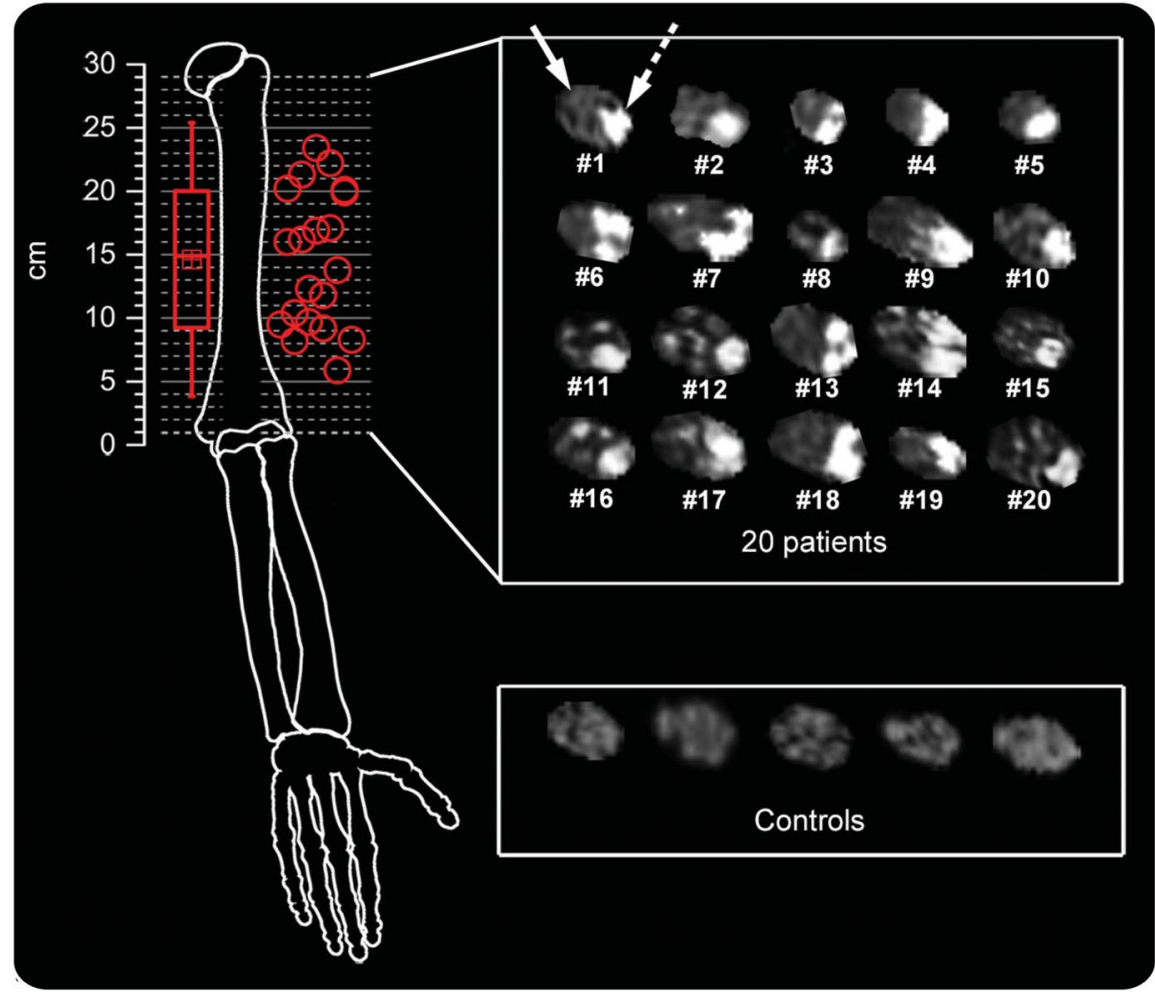

Spatial (red circles, upper left) and statistical (red box-and-whisker plot, upper left) distributions of predominant individual lesion sites and array of individual median nerve cross-sectional magnetic resonance neurography images (upper right) for the patient group ( $n=20$, numbered in accordance with table e-1). Localization of individual lesion sites is given as distance in $\mathrm{cm}$ from humeroradial joint space (at $0 \mathrm{~cm}$ ). Differences in individual arm lengths were not corrected for. In all patients, strongly increased T2 signal (bright) was found within a group of fascicles whereas other fascicles were spared, indicated by dashed (lesion) and solid (normal fascicles) white arrows in patient 1. Nerve T2 signal was normal in controls (lower right showing 5 representative out of 20 control subjects). 
topographic internal arrangement of a particular group of motor fascicles within the median nerve trunk at upper arm level: this fascicle group will form the AIN, which does not emerge from the median nerve trunk epineurium until further distally at forearm level.

Spatial lesion patterns and lesion extension. The longitudinal pattern of fascicular lesions was rated monofocal for patients 1 and 2. For patient 3, no agreement was obtained (indeterminate). Patients 4-20 were rated multifocal (Cohen kappa $=0.83, p<0.001$ ). Figure 3 illustrates the 2 different longitudinal lesion patterns on contiguous slices. Patients with monofocality were not discernable from patients with multifocality by presence of painful symptoms, type of onset, or by other clinical/electrophysiologic findings (table e-1). In none of the patients did T2 lesions extend to the proximal extreme of coverage (axilla).

Quantitative analysis of fascicular median nerve lesions. The mean normalized median nerve T2 value of 20 controls was $\mathrm{T} 2$ median_control $=1.19 \pm 0.05$. A similar value was found in patients for normal-appearing median nerve fascicles: T2 2 median_no_lesion $=1.39 \pm$ $0.08(p=0.104)$. In patients, however, the mean $\mathrm{T} 2_{\text {median_lesion }}=2.57 \pm 0.13$ of lesioned fascicles within the median nerve trunk was significantly higher compared with controls ( $\mathrm{T} 2$ median_lesion vs $\mathrm{T} 2$ median_control $; p<0.0001)$; it was also significantly higher compared with normal-appearing fascicles of patients $\left(\mathrm{T} 2_{\text {median_lesion }}\right.$ vs $\mathrm{T} 2_{\text {median_no_lesion; } p<}$
0.0001). Receiver operating characteristic (ROC) analysis of $\mathrm{T} 22_{\text {median_lesion }} \mathrm{vs} \mathrm{T} 22_{\text {median_control }}$ calculated an area under the curve (AUC) of 1.00 (95\% confidence interval [CI] 1.00-1.00), corresponding to sensitivity and specificity of $100 \%$ at a cutoff of $\geq 1.7$ of normalized T2 signal. ROC analysis of $\mathrm{T} 2$ median_lesion vs $\mathrm{T} 2$ median_no_lesion (lesioned fascicles vs normal-appearing fascicles of patients) revealed AUC $=0.98$ (95\% CI 0.94-1.00). Empirical values of normalized T2 signal for lesioned fascicles of patients are plotted against controls in figure 4.

Follow-up. In the majority of patients with multifocality, the administration of corticosteroids was elected as primary intervention. Their clinical response varied widely, with satisfactory recovery occurring in only some patients (table e-1). IV immunoglobulins (IVIg) were available for 4 patients, none of whom responded to corticosteroids, and were administered according to the ICE Study scheme. ${ }^{10}$ Upon IVIg, satisfactory improvement was observed in 2 of these 4 patients.

In patient 1 with monofocality, symptoms persisted after corticosteroids had been administered over 1 month (FPL 0). The monofocal proximal T2 lesion was discussed with the patient as a novel finding of unclear significance. It was mentioned that few cases had been described with torsion of motor median nerve fascicles at upper arm level, and that interfascicular neurolysis in some cases was followed by

Figure 2 Somatotopy of fascicular T2 median nerve lesion on individual level, group level, and atlas

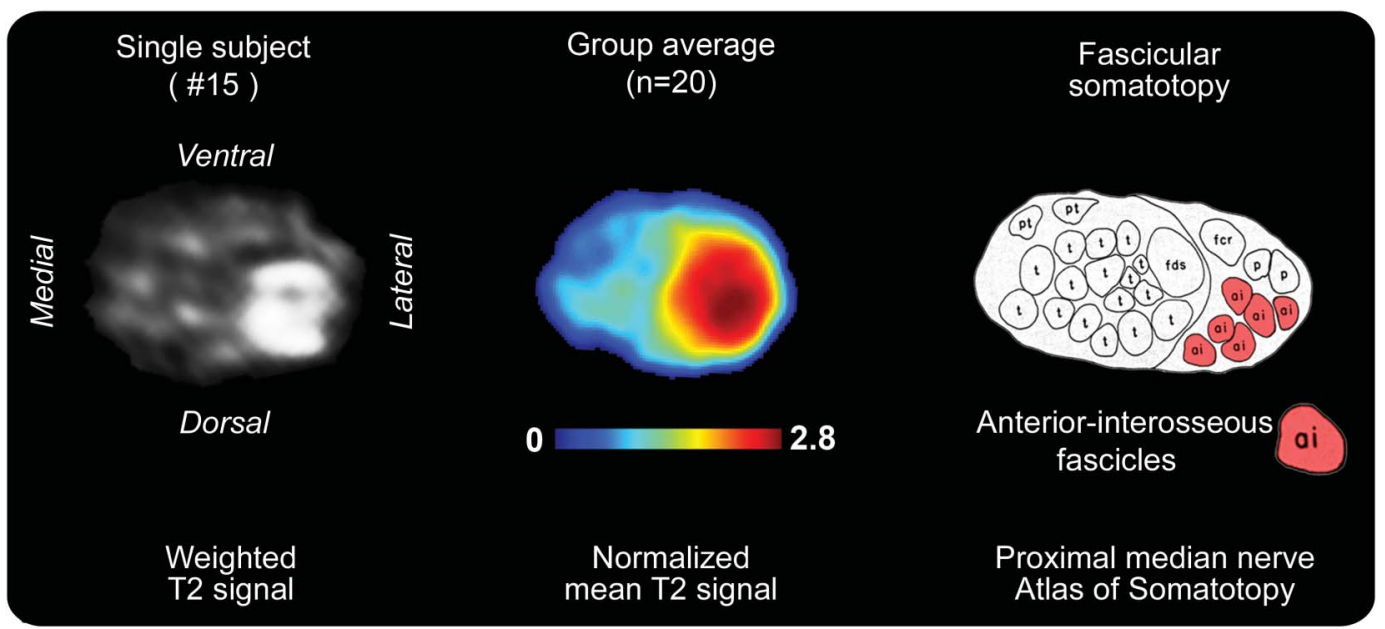

On the left, the T2-weighted source image of the median nerve of patient 15 is shown for the site of predominant lesion focus (17.1 cm proximal to humeroradial joint). Anatomical orientation is given by labeling ventral/dorsal/medial/lateral contours. In the middle, a spatial map of the patient group mean normalized T2 signal is shown. This map was rendered after segmentation and intersubject image registration. On the right is a somatotopic/topographic internal map of fascicles of the median nerve trunk. This schematic drawing was obtained by Jabaley et al. ${ }^{31}$ from tracing extraneural median nerve branches from distally to intraneural proximal fascicles within the median nerve trunk on 20 - $\mu \mathrm{m}$-thick cuts after intraneural microsurgical dissection and histologic photographing (modified from Jabaley et al. ${ }^{31}$ with permission). On this map, the red fascicles ("ai": anterior-interosseous) are in close spatial arrangement with the T2 lesion focus on individual (left) and group level (middle). This cross-sectional lesion area is at the dorsal and lateral/radial aspect of the median nerve at upper arm level with a mean distance of $14.6 \pm 5.4 \mathrm{~cm}$ proximal to the humeroradial joint space. 
Figure $3 \quad$ Monofocality and multifocality as 2 principal lesion patterns of anterior interosseous nerve syndrome

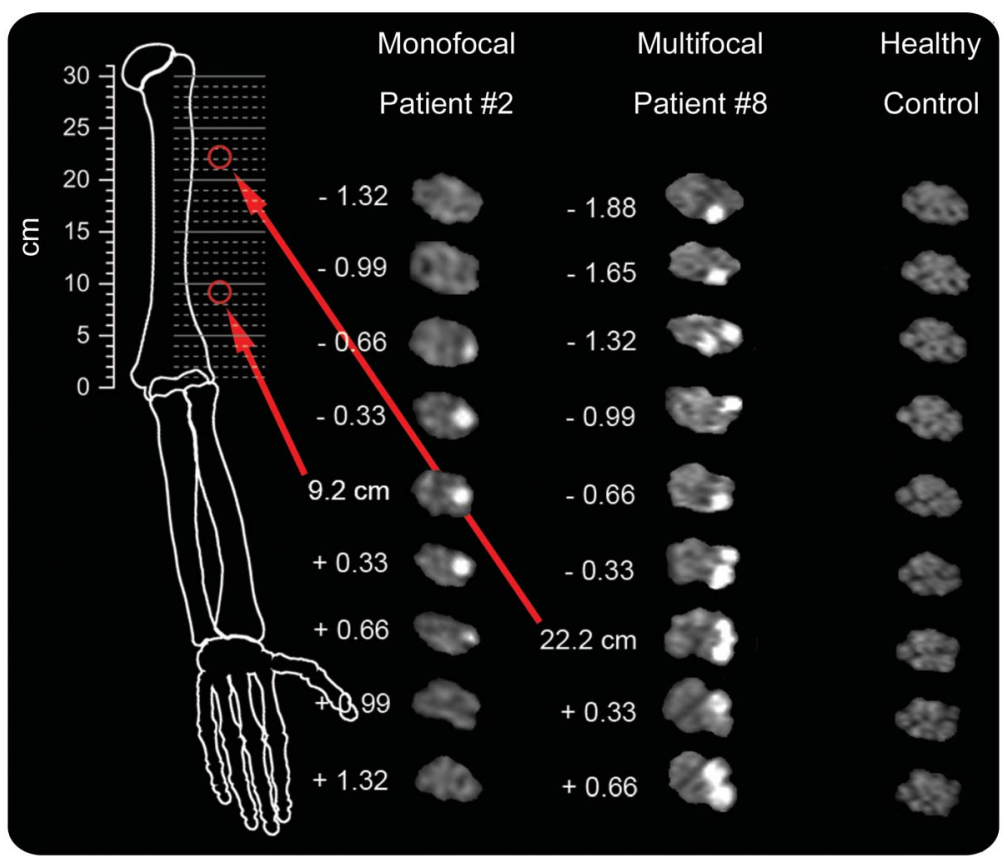

Representative monofocal (left) and multifocal (middle) lesion patterns are compared to one representative control subject (right). In each column, contiguous longitudinal high-resolution imaging slices are displayed. Numbers on the left of patient images indicate slice position relative to the predominant lesion focus (at $9.2 \mathrm{~cm}$ in patient 2 and $22.2 \mathrm{~cm}$ in patient 8). The monofocal and multifocal lesion pattern both follow the internal topography/somatotopy of anterior interosseous motor fascicles. These fascicles will form the anterior interosseous nerve further distally but at lesion site are located within epineurium of median nerve trunk. The anatomical localization of the predominant lesion focus is shown on the metric scale to the left of the schematic drawing of bony landmarks. Differences in individual arm lengths were not corrected for.

improvement. ${ }^{11-13}$ The patient opted for this surgical procedure. Epineurotomy was performed at the site of T2 lesion. Markedly swollen fascicles at the dorsal and lateral aspects of median nerve emerged with torsion around their longitudinal axis (figure 5). Careful mobilization and detorsion was performed. Follow-up until 8 months did not reveal any improvement and showed persistence of denervation with lack of voluntary activity in EMG of FPL and PQ. After 8 months, a positive Hoffmann-Tinel sign at the mid-forearm level was noticed. At 15 months, recovery to MRC 4 (from 0 ) was noted for FPL. In the second patient (2) with monofocality, administration of corticosteroids was paralleled by satisfactory improvement.

DISCUSSION The internal longitudinal organization of peripheral nerve fascicles was first studied in comprehensive fashion by Sunderland, ${ }^{14}$ who used surgical dissection to trace fascicles from distal to proximal. In this work, he described some degree of plexiform exchange between fascicles at proximal nerve levels. Other authors illustrated this finding as "intraneural chaos," ${ }^{15}$ which reflects that a concept of fascicular somatotopy-the meaningful grouping of nerve fascicles with regard to their function-has long been negated for the peripheral nervous system. ${ }^{16}$ Later, when the longitudinal course of fascicles became traceable on histology, it could be established that somatotopy is well-preserved despite some plexiform exchange between fascicles. ${ }^{15}$ The clinical implications of fascicular somatotopy have been reviewed in detail by Stewart, ${ }^{16}$ who emphasized that fascicular nerve lesions represent a major pitfall for lesion localization: typical symptom patterns that resemble the functional territory of a peripheral nerve trunk appear only if all fascicles at the lesion site are compromised. However, selective fascicular injury may result in symptoms not following expected distributions. For example, if only certain fascicles supplying distal muscles are selectively injured at a more proximal site, the lesion would be expected erroneously to involve a further distal nerve branch to this muscle group. To prove the existence of fascicular nerve lesions has been challenging because it is difficult if not impossible to localize and objectify these lesions by clinical/electrophysiologic findings.

In the case of AINS, the view of a neuropathy of the AIN itself at forearm level, or its terminal branches, prevails especially among authors from surgical disciplines. Consequently, entrapment, e.g., by a fibrous band, has been favored as principal mechanism and surgical release at forearm level advocated. ${ }^{17-24} \mathrm{Com}-$ peting views see AINS not as entrapment neuropathy, but as a disease of immune-mediated inflammatory origin. ${ }^{1,4,5,25}$ Certain similarities with neuralgic shoulder amyotrophy support an immune-mediated etiology. 5 Six of the original 136 patients reported by Parsonage and Turner $^{5}$ had weakness of FPL and FDPII, one of them without weakness of the shoulder girdle. Later, England and Sumner ${ }^{25}$ raised awareness that definite lesion localization remains difficult in Parsonage-Turner syndrome. From the distribution of symptoms in 9 well-documented cases, they concluded that lesion sites involve peripheral nerve branches rather than the brachial plexus and suspected involvement of the AIN in 4 patients. ${ }^{25}$

Improved lesion localization and determination of spatial lesion patterns would permit us to better understand the etiology of AINS and, in particular, to understand if and at which anatomical site AINS is potentially treatable by surgery. We prospectively investigated a relatively large sample of 20 patients with AINS and obtained detailed clinical/electrophysiologic data.

At the core of our study was MRN, providing large longitudinal coverage including upper arm, elbow, and forearm levels. With this protocol we sought to determine lesion sites and longitudinal lesion patterns by $\mathrm{T} 2$ signal analysis of median nerve fascicles. Increased T2 signal has been shown to indicate nerve injury of mechanical and nonmechanical origin, e.g., 


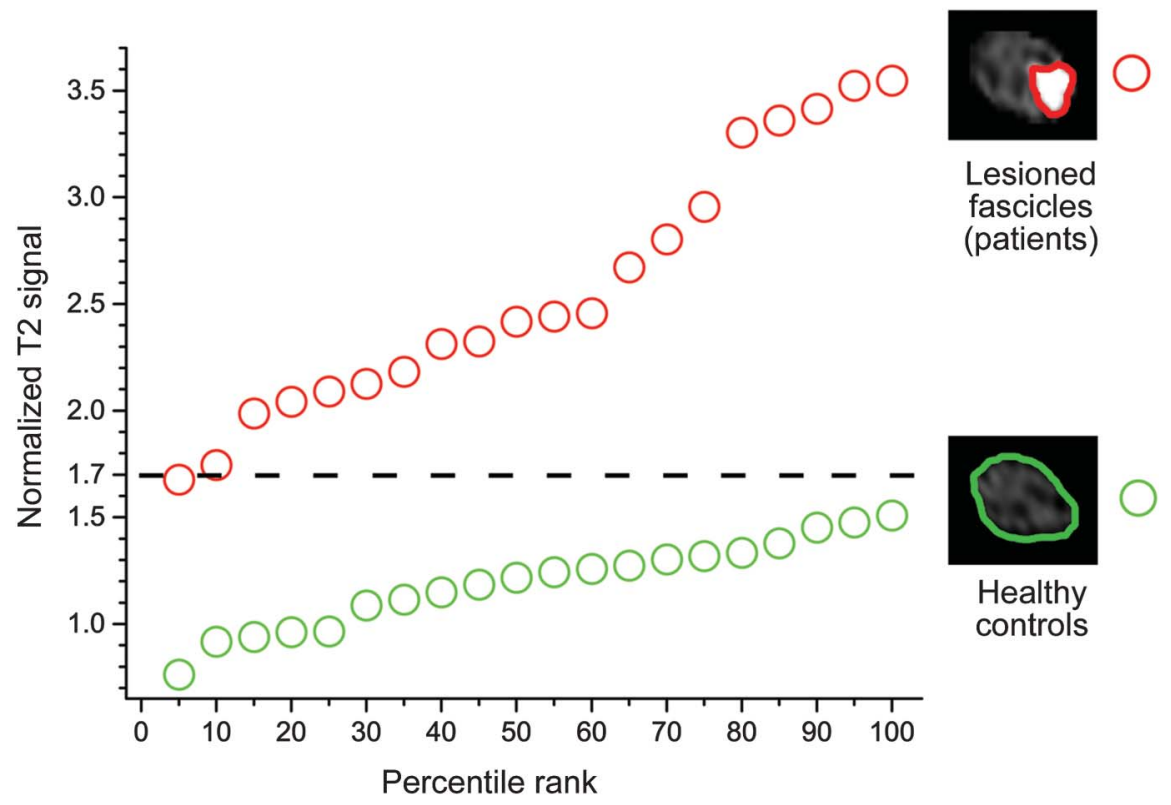

The upper graph (red) visualizes ordered percentile ranks of normalized T2 signal of each individual fascicular median nerve T2 lesion of the patient group ( $n=20$, red circles). The lower graph (green circles) visualizes percentile ranks of median nerve normalized T2 signal of the control group $(n=20)$. Perfect discriminative power to separate patients with anterior interosseous nerve syndrome from controls was observed at a cutoff value of $\geq 1.7$ of normalized T2 signal. The complete separation of both distributions corresponds to $100 \%$ sensitivity and $100 \%$ specificity. Manual segmentation of lesioned fascicles (patients) and normal median nerves of healthy controls was performed as indicated by red and green regions of interest on the right side of the diagram.

in focal entrapment, ${ }^{26,27}$ after trauma, ${ }^{28}$ in multifocal motor neuropathy, ${ }^{29}$ and also in metabolic polyneuropathies such as diabetic polyneuropathy. ${ }^{30}$

In AINS, we consistently found a strictly organized somatotopic/topographic internal fascicular lesion pattern within the median nerve at upper arm level: affected were those motor fascicles forming the AIN, which exits from the median nerve trunk further distally at forearm level. Other median nerve fascicles seemed to be spared. Our interpretation that this fascicular T2 lesion pattern corresponds to an exclusive or, at least, predominant involvement of the motor fascicles forming the AIN is based on its close resemblance to the position of the anterior interosseous fascicles as mapped by Jabaley et al. ${ }^{31}$ and on visual tracing of T2 lesion fascicles from proximally within the median nerve trunk to distally into the AIN itself, which was reliably recognizable at the given spatial resolution. We acknowledge limitations of both methods, e.g., interindividual variability and potential inaccuracy of visual rating. Therefore, we propose as future research aim to track lesion fascicles from proximal to distal by diffusion tensor imaging (DTI). However, so far, nerve DTI has not been implemented in humans at the submillimeter isotropic resolution needed to resolve fascicles. We further acknowledge that we cannot answer whether the extent of fascicular involvement differed between patients with complete and incomplete AINS, because it was beyond the limit of spatial resolution to determine the exact number of involved fascicles. It is noteworthy that fascicular lesions in some cases of AINS were detectable also by high-resolution ultrasound; however, lesion contrast and thus diagnostic performance of fascicular hypoechogenicity seem to be inferior to nerve fascicle increase of T2 signal (figure e-2).

In addition, the longitudinal lesion pattern was analyzed on contiguous slices. Multifocality was found in the majority of patients and monofocality in only 2 . Interestingly, there were no differences between these 2 distinct lesion patterns with regard to symptoms or clinical/electrophysiologic findings. The responses to therapeutic intervention were markedly heterogeneous in patients with both lesion patterns. This observation is in accordance with reported variable outcomes after therapeutic intervention in AINS and also with evidence that spontaneous recovery may occur in a substantial portion of patients. ${ }^{18,22,32}$

Monofocality in our cohort was rare. In one patient, surgical exploration with dissection of median nerve trunk epineurium (epineurotomy) revealed fascicular torsion precisely at the lesion site, which was localized by imaging (figure 5). After interfascicular neurolysis and detorsion, clinical recovery was observed in this patient. The rationale to offer such individually tailored surgical therapy guided by a novel imaging 


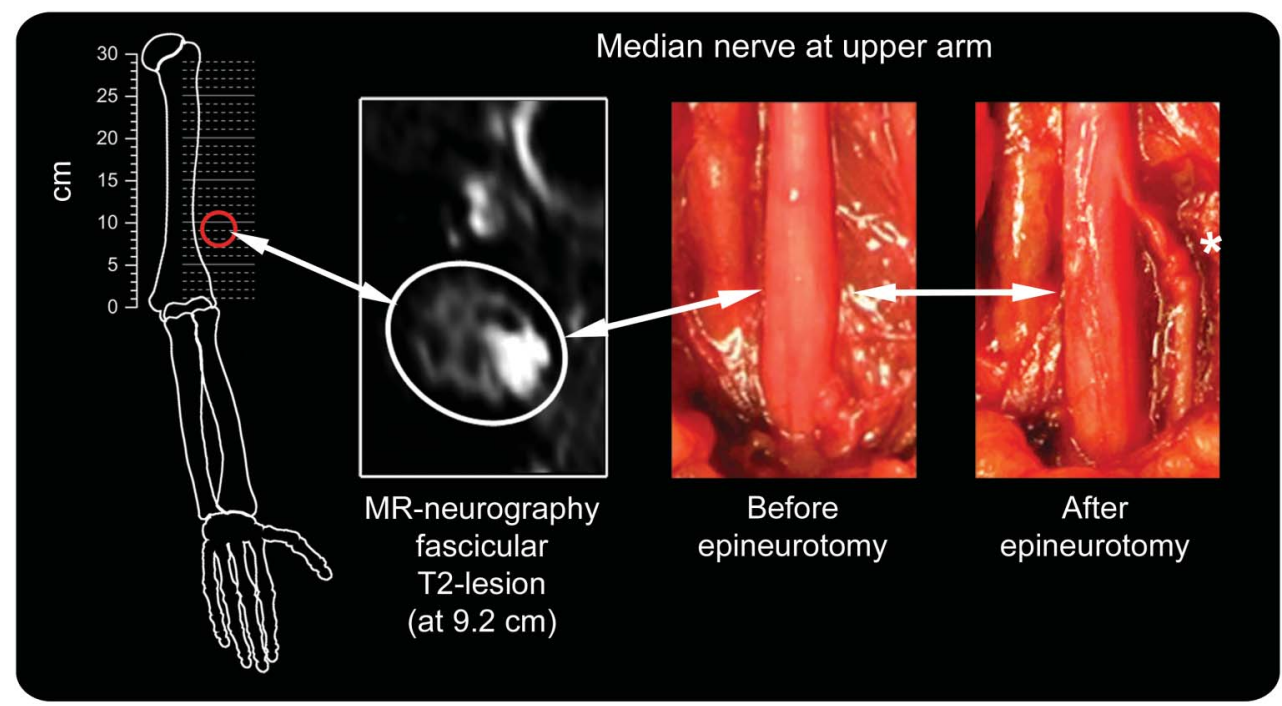

Monofocal T2 lesion on high-resolution magnetic resonance (MR) neurography of patient 1 guided targeted surgical epineurotomy of the median nerve trunk at upper arm level. The fascicular lesion focus (MR neurography fascicular T2 lesion) was at $9.2 \mathrm{~cm}$ proximal to the humeroradial joint space (left, red circle). Surgical exploration precisely at this level revealed subtle increase in median nerve trunk caliber before dissection of its epineurium (before epineurotomy, middle). Fascicular torsion of the anterior interosseous fascicles within the median nerve trunk was found after epineurotomy (asterisk, right). This constriction was carefully mobilized so that eventual detorsion could be performed, followed by satisfactory clinical recovery. Intraoperative images provided by Dr. Thomas Dombert, Dossenheim-Heidelberg, Germany.

sign was based on several case reports of median nerve fascicular torsion. ${ }^{11-13,33}$ Nagano and colleagues $^{11,33}$ reported the largest series to date and described an "hourglass-like fascicular constriction" between 2 and $7.5 \mathrm{~cm}$ above the elbow in 22 patients. Nagano ${ }^{33}$ reported good recovery after interfascicular neurolysis in 21 of 22 patients but stressed that "we do not know whether this recovery was spontaneous or due to the neurolysis." Fascicular torsion as a causative factor for AINS will remain difficult to prove. Some plausible explanations for its occurrence have been offered, particularly high mobility of AIN fascicles promoting torsion during elbow flexion, ${ }^{13}$ or initial inflammation and edema followed by intraneural adhesions, which increase traction forces on anterior interosseous fascicles. ${ }^{33}$

The histopathologic alterations underlying the observed T2 lesions remain unclear because biopsies at the lesion site are unethical. It is also difficult to explain by which pathophysiologic mechanisms proximal lesions are associated with functional compromise. It seems attractive to speculate that the accumulation of multifocal proximal injury is involved in the manifestation of symptoms and may result in further distally located functional or structural compromise, as believed to occur in other neuropathies such as diabetic polyneuropathy ${ }^{30,34,35}$ or the noncompressive polyneuropathy associated with type 2 neurofibromatosis. ${ }^{36}$

The significance of our results may be 2 -fold. This study is the first to provide strong diagnostic evidence by imaging for the existence of fascicular/partial nerve lesions in a spontaneously occurring neuropathy. The existence of fascicular nerve lesions had been assumed before but could not be objectified so far by NCS/ EMG studies. ${ }^{6,16}$ Invasive near-nerve recordings or even intraneural fascicular stimulation by needle microneurography would be necessary to detect selective fascicular conduction abnormalities. ${ }^{37,38}$ Both techniques are not readily available in humans and have not been reported in AINS. Noninvasive stimulation with surface electrodes for motor or sensory NCS typically remain normal and therefore nonlocalizing in AINS. EMG detects denervation in muscles supplied by the AIN; however, this finding is nonlocalizing: it cannot discriminate between injury to the AIN itself and a more proximal lesion of anterior interosseous fascicles within the median nerve trunk.

As second major implication, the predominance of lesions at upper arm level in all patients strongly supports that AINS is not an entrapment neuropathy of the AIN itself nor of its branches, at least in our cohort. The observation of selective fascicular lesions following motor somatotopy clearly suggests that AINS is a motor fascicular neuropathy of the median nerve trunk. The observation of multifocality in the majority of patients argues in favor of an immunemediated inflammatory origin and against any surgical treatment options either at forearm or upper arm level, at least in these multifocal cases. 


\section{AUTHOR CONTRIBUTIONS}

Dr. Pham: study design, analysis and interpretation of data, acquisition of data, writing of manuscript. Dr. Bäumer: acquisition of data, interpretation of data, revising manuscript for intellectual content. Dr. Meinck: acquisition of data, interpretation of data, revising manuscript for intellectual content. Dr. Schiefer: interpretation of data, revising manuscript for intellectual content. Dr. Weiler: interpretation of data, revising manuscript for intellectual content. Dr. Bendszus: study supervision, study design, interpretation of data, revising manuscript for intellectual content. Dr. Kele: study design, acquisition of data, analysis and interpretation of data, writing of manuscript.

\section{ACKNOWLEDGMENT}

The authors thank Dr. Thomas Dombert for documentation of the intraoperative field of patient 1 and for his permission to use the 2 intraoperative photographs of figure 5; and Prof. Dr. Sabine Heiland, Division of Experimental Radiology, Department of Neuroradiology, Heidelberg University Hospital, for assistance in setting up the MRN protocol and help in revising the manuscript.

\section{STUDY FUNDING}

This study was funded by a project grant from the German Osteoarthritis Foundation (P215-A482) and supported by a postdoctoral fellowship from the Medical Faculty of Heidelberg University (P.B.).

\section{DISCLOSURE}

M. Pham receives project grants from the German Osteoarthritis Foundation (Deutsche-Arthrose-Hilfe e.V.: P215-A482) and the EFSD/ JDRF/Novo Nordisk European Programme in Type 1 Diabetes Research. P. Bäumer receives a postdoctoral fellowship from the Medical Faculty of Heidelberg University, Germany. H. Meinck, J. Schiefer, and M. Weiler report no disclosures. M. Bendszus receives a project grant from the German Osteoarthritis Foundation (Deutsche-Arthrose-Hilfe e.V.: P215-A482). H. Kele reports no disclosures. Go to Neurology. org for full disclosures.

Received July 25, 2013. Accepted in final form October 10, 2013.

\section{REFERENCES}

1. Stevens JC. Median neuropathy. In: Dyck PJ, Thomas PK, eds. Peripheral Neuropathy, vol 1, 2, 4th ed. Philadelphia: Elsevier; 2005:1453-1454.

2. von Lanz T, Wachsmuth W. Praktische Anatomie: Arm, vol I/3, 2nd ed. Heidelberg: Springer; 1959.

3. Sunderland $\mathrm{S}$. The innervation of the flexor digitorum profundus and lumbrical muscles. Anat Rec 1945;93: 317-321.

4. Kiloh LG, Nevin S. Isolated neuritis of the anterior interosseous nerve. Br Med J 1952;1:850-851.

5. Parsonage MJ, Turner JW. Neuralgic amyotrophy: the shoulder-girdle syndrome. Lancet 1948;1:973-978.

6. Wertsch JJ, Sanger JR, Matloub HS. Pseudo-anterior interosseous nerve syndrome. Muscle Nerve 1985;8:68-70.

7. Wertsch JJ. AAEM case report \#25: anterior interosseous nerve syndrome. Muscle Nerve 1992;15:977-983.

8. Kimura J. Peripheral Nerve Diseases: Handbook of Clinical Neurophysiology, 1st ed. Philadelphia: Elsevier; 2006.

9. Smith SM, Jenkinson M, Woolrich MW, et al. Advances in functional and structural MR image analysis and implementation as FSL. Neuroimage 2004;23(suppl 1): S208-219.

10. Hughes RA, Donofrio P, Bril V, et al. Intravenous immune globulin ( $10 \%$ caprylate-chromatography purified) for the treatment of chronic inflammatory demyelinating polyradiculoneuropathy (ICE Study): a randomised placebocontrolled trial. Lancet Neurol 2008;7:136-144.
11. Nagano A, Shibata K, Tokimura H, et al. Spontaneous anterior interosseous nerve palsy with hourglass-like fascicular constriction within the main trunk of the median nerve. J Hand Surg Am 1996;21:266-270.

12. Yasunaga $\mathrm{H}$, Shiroishi $\mathrm{T}$, Ohta K, et al. Fascicular torsion in the median nerve within the distal third of the upper arm: three cases of nontraumatic anterior interosseous nerve palsy. J Hand Surg Am 2003;28:206-211.

13. Haussmann P, Patel MR. Intraepineurial constriction of nerve fascicles in pronator syndrome and anterior interosseous nerve syndrome. Orthop Clin North Am 1996;27: 339-344.

14. Sunderland S. Nerves and Nerve Injuries. Edinburgh: Churchill Livingstone; 1978.

15. Brushart TM. Central course of digital axons within the median nerve of Macaca mulatta. J Comp Neurol 1991; 311:197-209.

16. Stewart JD. Peripheral nerve fascicles: anatomy and clinical relevance. Muscle Nerve 2003;28:525-541.

17. Mackinnon SE, Dellon AL. Surgery of the Peripheral Nerve. New York: Thieme; 1988.

18. Lake PA. Anterior interosseous nerve syndrome. J Neurosurg 1974;41:306-309.

19. Spinner M. The anterior interosseous nerve syndrome, with special attention to its variations. J Bone Joint Surg Am 1970;52:84-94.

20. Fearn CB, Goodfellow JW. Anterior interosseous nerve palsy. J Bone Joint Surg Br 1965;47:91-93.

21. Farber JS, Bryan RS. The anterior interosseous nerve syndrome. J Bone Joint Surg Am 1968;50:521-523.

22. Vichare NA. Spontaneous paralysis of the anterior interosseous nerve. J Bone Joint Surg Br 1968;50:806-808.

23. Collins DN, Weber ER. Anterior interosseous nerve syndrome. South Med J 1983;76:1533-1537.

24. Nigst H, Dick W. Syndromes of compression of the median nerve in the proximal forearm (pronator teres syndrome; anterior interosseous nerve syndrome). Arch Orthop Trauma Surg 1979;93:307-312.

25. England JD, Sumner AJ. Neuralgic amyotrophy: an increasingly diverse entity. Muscle Nerve 1987;10: 60-68.

26. Baumer P, Dombert T, Staub F, et al. Ulnar neuropathy at the elbow: MR neurography-nerve T2 signal increase and caliber. Radiology 2011;260:199-206.

27. Subhawong TK, Wang KC, Thawait SK, et al. High resolution imaging of tunnels by magnetic resonance neurography. Skeletal Radiol 2012;41:15-31.

28. Filler AG, Howe FA, Hayes CE, et al. Magnetic resonance neurography. Lancet 1993;341:659-661.

29. Van Asseldonk JT, Franssen H, Van den Berg-Vos RM, et al. Multifocal motor neuropathy. Lancet Neurol 2005; 4:309-319.

30. Pham M, Oikonomou D, Baumer P, et al. Proximal neuropathic lesions in distal symmetric diabetic polyneuropathy: findings of high-resolution magnetic resonance neurography. Diabetes Care 2011;34:721-723.

31. Jabaley ME, Wallace WH, Heckler FR. Internal topography of major nerves of the forearm and hand: a current view. J Hand Surg Am 1980;5:1-18.

32. Huffmann G, Leven B. N. interosseous anterior syndrome: study in 4 cases of our own and in 49 cases from the literature (author's transl) [in German]. J Neurol 1976; 213:317-326. 
33. Nagano A. Spontaneous anterior interosseous nerve palsy. J Bone Joint Surg Br 2003;85:313-318.

34. Dyck PJ, Karnes JL, O'Brien P, et al. The spatial distribution of fiber loss in diabetic polyneuropathy suggests ischemia. Ann Neurol 1986;19:440-449.

35. Dyck PJ, Lais A, Karnes JL, et al. Fiber loss is primary and multifocal in sural nerves in diabetic polyneuropathy. Ann Neurol 1986;19:425-439.
36. Baumer P, Mautner VF, Baumer T, et al. Accumulation of non-compressive fascicular lesions underlies NF2 polyneuropathy. J Neurol 2013;260:38-46.

37. Hallin RG, Wu G. Fitting pieces in the peripheral nerve puzzle. Exp Neurol 2001;172:482-492.

38. Hallin RG. Microneurography in relation to intraneural topography: somatotopic organisation of median nerve fascicles in humans. J Neurol Neurosurg Psychiatry 1990;53:736-744.

\section{www.neurology.org Offers Important Information to Patients and Their Families}

The Neurology ${ }^{\circledR}$ Patient Page provides:

- A critical review of ground-breaking discoveries in neurologic research that are written especially for patients and their families

- Up-to-date patient information about many neurologic diseases

- Links to additional information resources for neurologic patients

All Neurology Patient Page articles can be easily downloaded and printed, and may be reproduced to distribute for educational purposes. Click on the 'Patients' link on the home page (www.neurology.org) for a complete index of Patient Pages.

\section{Earn 20 CME Credits Toward MOC with New NeuroPI ${ }^{\mathrm{SM}}$ Modules}

Choose from the latest lineup of quality modules to join the AAN's exclusive performance improvement programs designed to help you address both the Performance in Practice (PIP) and Continuing Medical Education (CME) components of Maintenance of Certification (MOC).

- NEW! Distal Symmetric Polyneuropathy (DSP) includes eight quality measures, addressing accurate and appropriate evaluation/monitoring of DSP and associated symptoms to guide treatment options, patient safety, and best practices to assist patients in managing their pain and improving quality of life

- Acute Stroke addresses six quality measures, including deep vein thrombosis prophylaxis (DVT) for ischemic stroke or intracranial hemorrhage, discharged on antiplatelet therapy, dysphagia screening, rehabilitation service considerations, and more

- Dementia includes 10 quality measures addressing underuse of effective services and patientcentered care strategies, and patient safety issues

Learn about all of the other available modules and purchase yours today:

www.aan.com/view/neuropi 


\section{Neurology}

Anterior interosseous nerve syndrome: Fascicular motor lesions of median nerve trunk Mirko Pham, Philipp Bäumer, Hans-Michael Meinck, et al.

Neurology 2014;82;598-606 Published Online before print January 10, 2014

DOI 10.1212/WNL.0000000000000128

This information is current as of January 10, 2014

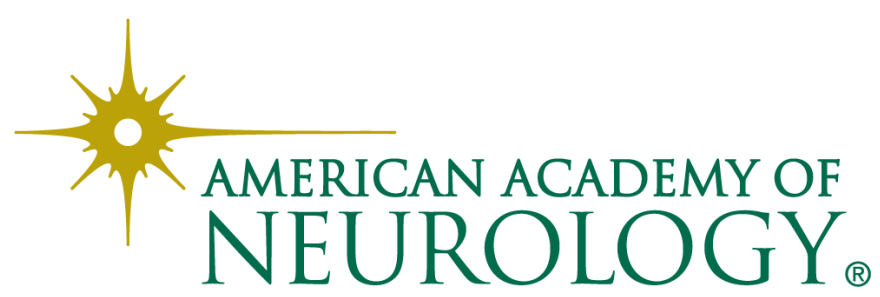




\section{Updated Information \& Services}

\section{Supplementary Material}

\section{References}

\section{Citations}

Subspecialty Collections

\section{Permissions \& Licensing}

\section{Reprints}

including high resolution figures, can be found at:

http://n.neurology.org/content/82/7/598.full

Supplementary material can be found at: http://n.neurology.org/content/suppl/2014/01/10/WNL.0000000000000 128.DC1

http://n.neurology.org/content/suppl/2014/01/10/WNL.0000000000000 128.DC2

http://n.neurology.org/content/suppl/2014/10/31/WNL.0000000000000 128.DC3

This article cites 34 articles, 3 of which you can access for free at: http://n.neurology.org/content/82/7/598.full\#ref-list-1

This article has been cited by 5 HighWire-hosted articles: http://n.neurology.org/content/82/7/598.full\#\#otherarticles

This article, along with others on similar topics, appears in the following collection(s):

\section{All Clinical Neurology}

http://n.neurology.org/cgi/collection/all_clinical_neurology

All clinical neurophysiology

http://n.neurology.org/cgi/collection/all_clinical_neurophysiology

All Neuromuscular Disease

http://n.neurology.org/cgi/collection/all_neuromuscular_disease

\section{MRI}

http://n.neurology.org/cgi/collection/mri

Peripheral neuropathy

http://n.neurology.org/cgi/collection/peripheral_neuropathy

Information about reproducing this article in parts (figures,tables) or in its entirety can be found online at:

http://www.neurology.org/about/about_the_journal\#permissions

Information about ordering reprints can be found online:

http://n.neurology.org/subscribers/advertise

Neurology ${ }^{\circledR}$ is the official journal of the American Academy of Neurology. Published continuously since 1951, it is now a weekly with 48 issues per year. Copyright @ 2014 American Academy of Neurology. All rights reserved. Print ISSN: 0028-3878. Online ISSN: 1526-632X.

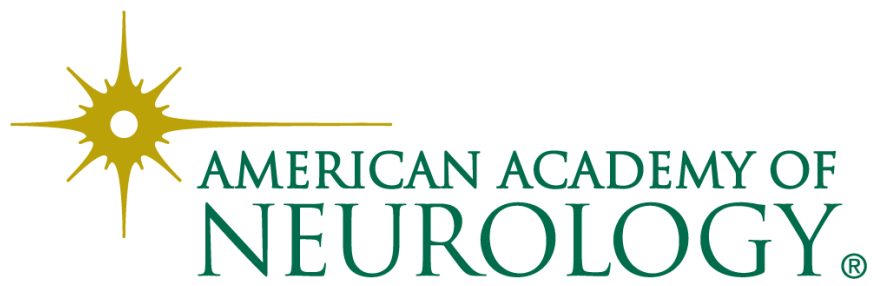

